1 Injury Epidemiology in Professional Ballet: A Five-

2 Season Prospective Study of 1596 Medical Attention

3 Injuries and 543 Time-Loss Injuries

4

5

6

7

8

Submission type: Original research

\title{
Authors:
}

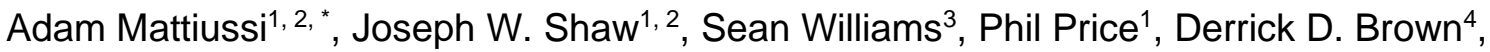

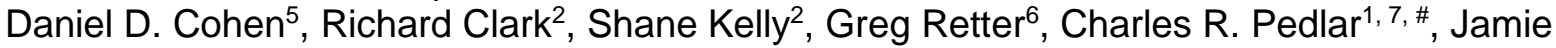
Tallent ${ }^{1, \#}$

\section{Institutional/Corporate affiliations:}

${ }^{1}$ Faculty of Sport, Allied Health and Performance Science, St Mary's University, Twickenham, UK

2 Ballet Healthcare, The Royal Ballet, The Royal Opera House, London, UK

${ }^{3}$ Sport Injury Prevention at Bath, Department for Health, University of Bath, Bath, UK

${ }^{4}$ Institute of Sport Science, Dance Science, University of Bern, Bern, Switzerland

${ }^{5}$ Faculty of Health Sciences, University of Santander, Bucaramanga, Colombia

${ }^{6}$ Team GB, British Olympic Association, London, UK

${ }^{7}$ Division of Surgery and Interventional Science, University College London, UK

\#Joint last author

Charles R. Pedlar and Jamie Tallent

"Correspondence:

Adam Mattiussi

Mattiussi.adam@gmail.com

Date Accepted for Publication: 31/03/21

Journal: British Journal of Sports Medicine

DOI: http://dx.doi.org/10.1136/bjsports-2020-103817 
ABSTRACT

Objectives To describe the incidence rate, severity, burden, and aetiology of medical attention and time-loss injuries across five consecutive seasons at a professional ballet company.

Methods Medical attention injuries, time-loss injuries, and dance exposure hours of 123 professional ballet dancers (female: $n=66$, age: $28.0 \pm 8.3$ y; male: $n=57$, age: $27.9 \pm 8.5$ y) were prospectively recorded between the $2015 / 16$ and $2019 / 20$ seasons.

Results The incidence rate (per $1000 \mathrm{~h}$ ) of medical attention injury was 3.9 (95\% Cl: $3.3-4.4)$ for females and 3.1 (95\% Cl: 2.6-3.5) for males. The incidence rate (per $1000 \mathrm{~h}$ ) of time-loss injury was $1.2(95 \% \mathrm{Cl}: 1.0-1.5)$ for females and $1.1(95 \% \mathrm{Cl}: 0.9-1.3)$ for males. First Soloists and Principals experienced between 2.0-2.2 additional medical attention injuries per 1000 hours and 0.9-1.1 additional time-loss injuries per 1000 hours compared to Apprentices ( $p \leq$ .025). Further, intra-season differences were observed in medical attention, but not time-loss, injury incidence rates with the highest incidence rates in early (August and September) and late (June) season months. Thirty-five percent of time-loss injuries resulted in over 28 days of modified dance training. A greater percentage of time-loss injuries were classified as overuse (female: $50 \%$; male: $51 \%$ ) compared to traumatic (female: $40 \%$; male: $41 \%$ ).

Conclusion This is the first study to report the incidence rate of medical attention and timeloss injuries in professional ballet dancers. Incidence rates differed across company ranks and months, which may inform targeted injury prevention strategies.

Key Words: Aetiology, Dance Medicine, Injury Surveillance 


\section{What are the new findings?}

- This is the first study to document medical attention incidence rate in professional ballet and identify the burden placed on dance medicine and science teams through non-time-loss musculoskeletal complaints.

- Time-loss and medical attention incidence rates are highest in First Soloists and Principal dancers of a professional ballet company.

- Medical attention injury incidence rates are greater during the start and the end of the season compared to mid-season.

- The severity of time-loss injuries is high, with $35 \%$ of all injuries resulting in more than 28 days of modified dance.

\section{How might it impact on clinical practice in the future?}

- Company rank and month of the season offer opportunity to target context-specific risk factors in professional ballet.

- Lower extremity injuries may be addressed by injury-specific prevention strategies. These strategies may include targeting the ankle in females, and stress fractures of the foot and tibia in males.

- A high proportion of injuries were overuse in nature. Improved management of the rehearsal and performance schedule may mitigate the burden of these injuries.

- A common mechanism of injury was jumping and landing activities, which may warrant further attention from dance science and medicine practitioners. 
Injury Epidemiology in Professional Ballet

\section{INTRODUCTION}

The probability of sustaining a musculoskeletal injury in professional ballet is high, with one article reporting an incidence proportion of 6.8 injuries per dancer over a season. ${ }^{1}$ However, differences in time-loss injury are observed across professional ballet companies, with incidence proportions ranging from 1.8-6.8 injuries per dancer. ${ }^{1-7}$ Similarly, differences in incidence rates are observed across studies, with values ranging from 0.6-4.4 injuries per 1000 hours of dance exposure..$^{1-3,5}$ The variation in incidence rates may reflect the use of contractual hours when calculating dance exposure (as opposed to individualised class, rehearsal, and performance schedules) or inconsistent injury definitions across studies. ${ }^{3-7}$

No research has described the incidence rate of medical attention injuries in professional ballet. The inclusion of medical attention injuries in epidemiology research has been recommended by Clarsen and $\mathrm{Bahr}^{8}{ }^{8}$ and various consensus statements in sport, ${ }^{9,10}$ as it provides a more comprehensive understanding of the medical burden within an organisation. Medical attention injuries, for example, impact performance outcomes in professional cricket. ${ }^{11}$ Although performance outcomes in professional ballet are less tangible than sport, medical attention injury incidence rates may affect casting. Quantifying the incidence rate of medical attention injuries alongside time-loss injuries is therefore an important step towards effective medical management within professional ballet. ${ }^{12}$

Most injury epidemiology research in professional ballet is not reported in line with current methodological standards and lacks comprehensive contextual detail. ${ }^{13}$ For example, atypical or no severity scales have been applied, there is inconsistent reporting of injury definitions, diagnoses, and tissue types, and few studies have reported differences in injury incidence rates and aetiology across contextual risk factors. ${ }^{1-7}$ Specific injury risk factors, such as sex, company rank, and intra- and inter-season variation, have been identified in professional ballet. ${ }^{14,15}$ However, only one study has reported statistical differences in injury incidence rates across sex and rank, ${ }^{1}$ and although several studies have reported longitudinal injury incidence rates in professional ballet dancers, ${ }^{2,3,5-7}$ none of these conduct statistical analyses. 
Injury Epidemiology in Professional Ballet

This study aimed to investigate the sex, company rank, and intra- and inter-season differences in medical attention and time-loss injury incidence rates across five consecutive seasons at a professional ballet company. We also aimed to describe the severity, burden, and aetiology of medical attention and time-loss injuries.

\section{METHODS}

\section{Study Design and Setting}

A prospective cohort study design was employed to investigate medical attention and timeloss injuries in professional ballet dancers. Data were collected across five consecutive seasons at The Royal Ballet, commencing August $8^{\text {th }} 2015$ and ending March $15^{\text {th }} 2020$. The 2019/20 season ended prematurely due to the COVID-19 global pandemic. All scheduled dance events were completed within the Royal Opera House, London. All dance exposure and medical data were entered into standardised electronic forms (Smartabase version 6.5.11, Fusion Sport, Brisbane, Australia). Medical attention and time-loss injuries were evaluated and recorded by in-house Chartered Physiotherapists, typically within 24 hours of the onset. Dance exposure data were prospectively entered by the company Artistic Scheduling Manager. Injury diagnoses were categorised using version 10 of the Orchard Sports Injury Classification System (OSICS). ${ }^{16}$ Data entered outside of each season were excluded from the analysis (e.g., tour, summer break). There was no patient or public involvement in the design, conduct, or reporting of this study.

\section{Participants}

Of 124 eligible elite professional dancers across the ranks of Apprentice, Artist, First Artist, Soloist, First Soloist, Principal, and Principal Character Artist, 123 were included in this analysis (female: 66 , age: $28.0 \pm 8.3$ y; male: 57 , age: $27.9 \pm 8.5$ y; Figure 1 ). Dancers who joined or left the company during the study period were included for the duration of their time 
Injury Epidemiology in Professional Ballet

130 in the company. Written informed consent was provided by 108 dancers. The remaining 16

131 were contacted, one of which declined consent, and 15 did not respond. A legitimate interest

132 assessment to use the anonymised data for the present analysis was approved by the Data

133 Controller of the Royal Opera House, in line with GDPR (2016) and the UK Data Protection

134 Act (2018). Written support was provided by the Clinical Director of The Royal Ballet. Ethical

135 approval was granted by St Mary's University Ethics Committee in accordance with the 136 Declaration of Helsinki. 
A

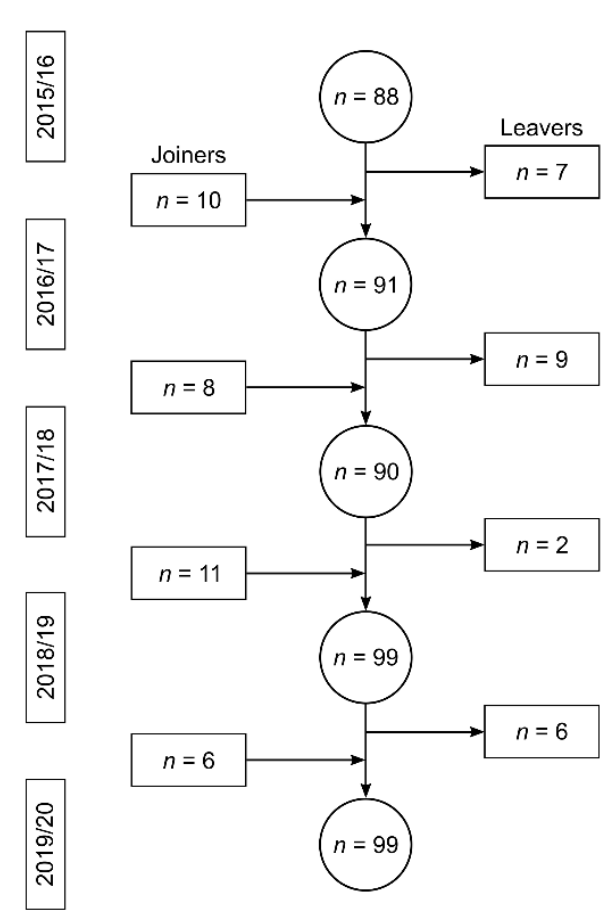

B

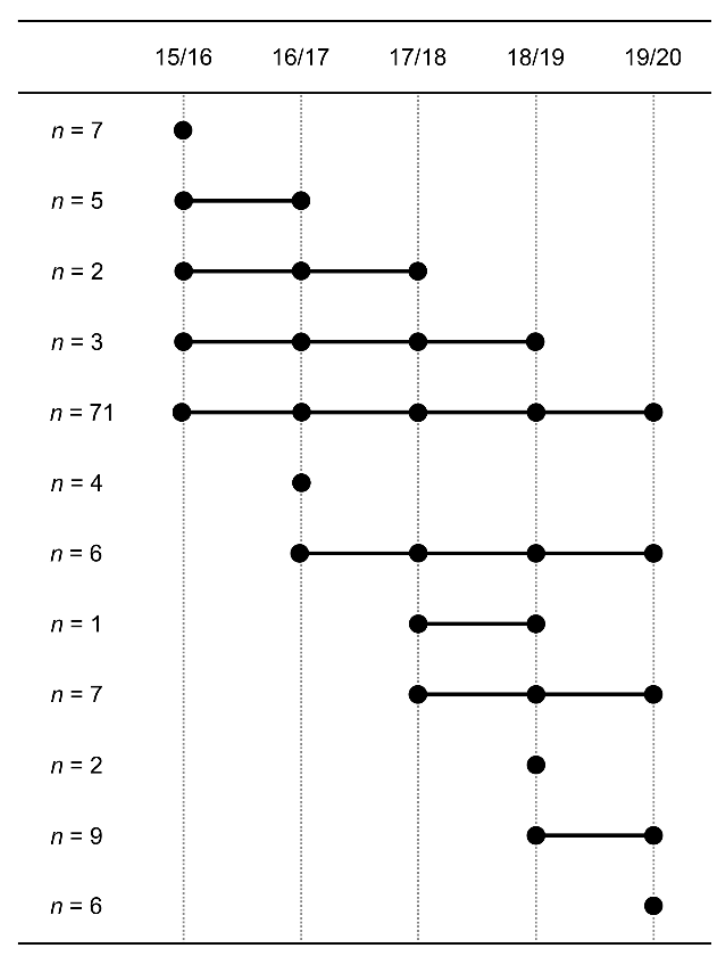

C

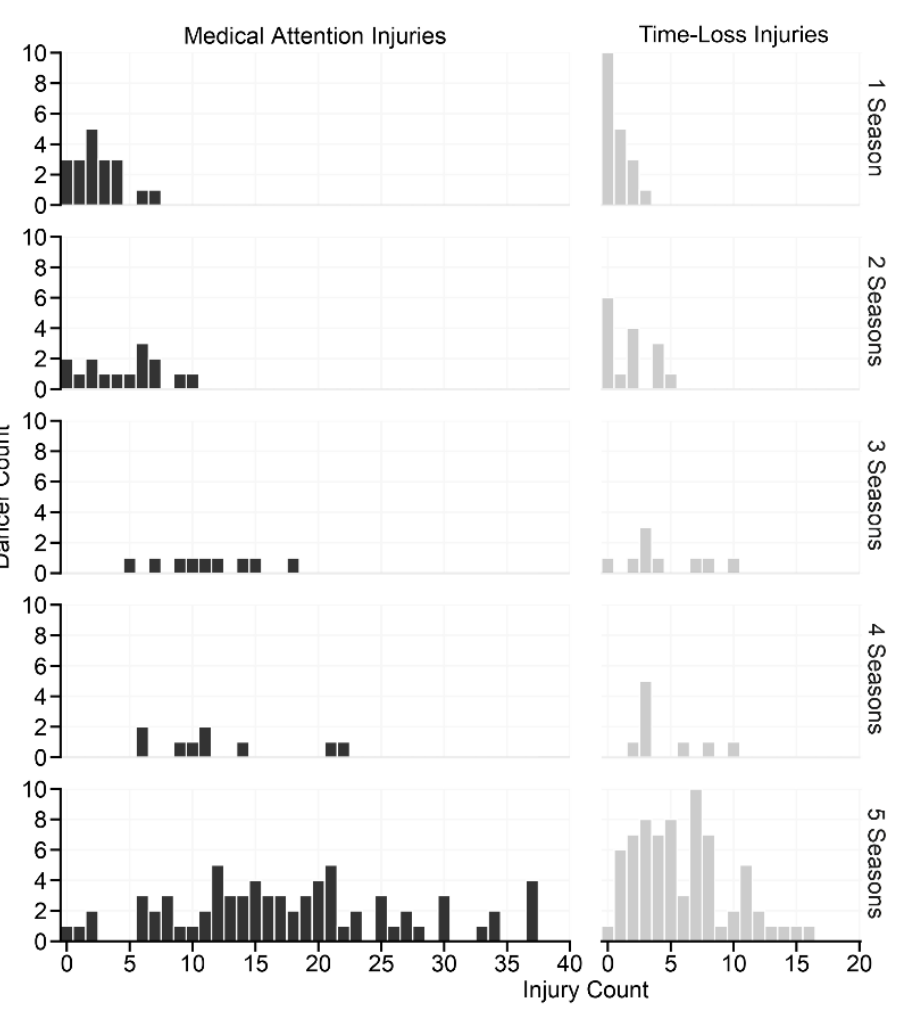

Figure $1 \mathrm{~A}$ ) The number of participants joining, leaving, and present each season. B) The number of participants who were present across 
Injury Epidemiology in Professional Ballet

\section{Injury Definitions}

Medical attention injuries were defined as "any musculoskeletal complaint that required medical attention from a physiotherapist". ${ }^{8}$ Time-loss injuries were defined as "any injury that prevented a dancer from taking a full part in all dance-related activities that would normally be required of them for a period equal to or greater than 24 hours after the injury was sustained". ${ }^{1}$ Time-loss injuries were closed on the date of their final appointment when no follow-up appointment occurred within 28 days. Prevalence was defined as the count of injured dancers divided by the count of included dancers each season. Incidence proportion was defined as the count of injuries divided by the count of included dancers each season. Severity was classified as either minor ( $1-7$ days), moderate (8-28 days), or severe ( $>28$ days).${ }^{17}$ Recurrent injury was defined as "any injury of the same location and type as the index injury, which occurred following a full return to all dance-related activities". ${ }^{18}$ Overuse injuries were defined as "any medical incident that did not have a sudden onset from a discrete event". ${ }^{19}$ The nature of injuries were categorised based on the physiotherapist's interpretation of the primary risk factor, where intrinsic was related to the characteristics of the individual and extrinsic was related to environmental factors. ${ }^{2}$ The term "not classified" was applied when a physiotherapist was unable to distinguish the mechanism, activity, footwear, classification, occurrence, or nature of the injury.

\section{Data Analysis}

\section{Dance Exposure}

Individualised exposure hours for class, rehearsal, and performance were extracted from the online data management system and calculated for each dancer. Performance casts for each show were inspected manually and cross-referenced with updated casting sheets to account for cast changes. Following a new time-loss injury, prospectively scheduled dance events were removed to accurately calculate dance exposure. Individualised rehearsal and performance exposure hours were grouped by production length (i.e., stand-alone full-length 
Injury Epidemiology in Professional Ballet

169

170

171

172

173

174

175

176

177

178

179

180

181

182

183

184

185

186

187

188

189

190

191

192

ballets ( $\geq 90$ minutes) or shorter productions that were staged together ( $<90$ minutes)), and by production type (i.e., new creations or existing works).

\section{Medical Attention and Time-Loss Injury}

The total medical attention injuries, time-loss injuries, and exposure hours were calculated for each unique dancer and grouped by sex, rank, month, season. The incidence rate (per 1000 h) of medical attention and time-loss injuries by production length, production type, anatomical region, and tissue type was calculated by dividing grouped injury count by grouped exposure time. Mean prevalence and incidence proportion of medical attention and time-loss injuries were calculated across the four complete seasons (2015/16-2018/19). Time-loss injury severity was calculated as median days lost, as severity data were not normally distributed. Time-loss injury severity was also calculated as the percentage of injuries classed as minor, moderate, and severe. Injury burden (days lost per $1000 \mathrm{~h}$ ) and risk matrices (incidence rate $x$ median severity) were calculated by anatomical region and tissue type. The number and percentage of medical attention and time-loss injuries by activity, mechanism, footwear, occurrence, classification, nature were calculated. For all values, 95\% confidence intervals (CI) were calculated. Mechanism of injury fields were concatenated based on movement similarities (e.g., 'Plié' and 'Relevé' became 'Plié/relevé'). The anatomical region and tissue type of injuries were classified using the OSICS diagnosis code. ${ }^{13,16}$ There were five open injury records at the onset of the study. Three dancers were partaking in restricted rehearsals, and were therefore included in the study from the onset. Two were fully removed from normal rehearsal, but returned to rehearsal after 34 and 55 days; these dancers were included in the study following their return. 
Injury Epidemiology in Professional Ballet

193

194

195

196

197

198

199

200

201

202

203

204

205

206

207

208

209

210

211

212

213

214

215

216

217

218

\section{Statistical Analysis}

A Poisson generalized linear mixed model was used to calculate incidence rates for all medical attention and time-loss injuries using the Ime4 package. ${ }^{20}$ The output variable was the number of recorded medical attention and time-loss injuries offset by the log of dance exposure hours for each individual. Sex, rank, sex $\times$ rank interaction, month, and season were included as fixed factors. Dancer identity was included as a random factor to account for repeated observations over time. Main effects of the generalized linear mixed model were compared by applying an analysis of variance using the car package. ${ }^{21}$ The estimated marginal means (EMM) for each fixed factor were extracted from the model, with $95 \% \mathrm{Cl}$, and backtransformed to calculate incidence rate per 1000 hours using the emmeans package. ${ }^{22}$ Posthoc pairwise comparisons, with false discovery rate adjustment, were used to investigate statistically significant main effects. ${ }^{22}$ Significance was set at $p \leq .025$ to account for two primary outcome measures. All data and statistical analysis were conducted using $R$ (version 4.0.3, R Foundation for Statistical Computing, Vienna, Austria).

\section{RESULTS}

\section{Dance Exposure}

There were 20,762 unique scheduled dance events over 5 consecutive seasons. This resulted in 283,453 individual dancer events (class: 99,733; rehearsal: 152,588; performance: 31,132). Scheduled dance events represented a total of 417,693 hours of individual dance exposure (class: 115,772; rehearsal: 209,529; performance: 92,392).

\section{Injuries}

Table 1 outlines the number of dancers, medical attention injuries, and time-loss injuries over the five seasons. The count of injuries by dancer and number of seasons in the company is presented in Figure 1 
Injury Epidemiology in Professional Ballet

219 Table 1 Number of dancers, medical attention injuries, and time-loss injuries across five consecutive seasons.

\begin{tabular}{|c|c|c|c|c|c|c|c|c|c|c|c|c|c|c|c|}
\hline & \multicolumn{3}{|c|}{$2015 / 16$} & \multicolumn{3}{|c|}{ 2016/17 } & \multicolumn{3}{|c|}{$2017 / 18$} & \multicolumn{3}{|c|}{ 2018/19 } & \multicolumn{3}{|c|}{$2019 / 20$} \\
\hline & $n$ & MA & $\mathrm{TL}$ & $n$ & MA & $\mathrm{TL}$ & $n$ & MA & TL & $n$ & MA & $\mathrm{TL}$ & $n$ & MA & $\mathrm{TL}$ \\
\hline All & 88 & 384 & 88 & 91 & 305 & 112 & 90 & 338 & 138 & 99 & 286 & 130 & 99 & 283 & 75 \\
\hline Female & 48 & 228 & 53 & 50 & 180 & 60 & 49 & 183 & 75 & 52 & 171 & 70 & 53 & 163 & 42 \\
\hline App. & 2 & 8 & 1 & 4 & 10 & 4 & 3 & 4 & 2 & 4 & 5 & 1 & 4 & 12 & 2 \\
\hline Artist & 11 & 46 & 8 & 11 & 46 & 11 & 10 & 53 & 21 & 14 & 53 & 17 & 12 & 33 & 7 \\
\hline F. Artist & 9 & 35 & 12 & 10 & 31 & 11 & 11 & 43 & 26 & 10 & 28 & 10 & 12 & 48 & 16 \\
\hline Soloist & 11 & 62 & 21 & 9 & 27 & 10 & 8 & 30 & 7 & 4 & 11 & 3 & 5 & 15 & 3 \\
\hline F. Soloist & 7 & 39 & 5 & 7 & 33 & 12 & 6 & 18 & 4 & 9 & 37 & 21 & 9 & 31 & 3 \\
\hline Principal & 6 & 36 & 6 & 8 & 31 & 10 & 8 & 30 & 12 & 8 & 29 & 13 & 8 & 16 & 8 \\
\hline PCA & 2 & 2 & 0 & 1 & 2 & 2 & 3 & 5 & 3 & 3 & 8 & 5 & 3 & 8 & 3 \\
\hline Male & 40 & 156 & 35 & 41 & 125 & 52 & 41 & 155 & 63 & 47 & 115 & 60 & 46 & 120 & 33 \\
\hline App. & 3 & 6 & 0 & 4 & 9 & 2 & 4 & 18 & 9 & 4 & 4 & 1 & 2 & 4 & 0 \\
\hline Artist & 7 & 30 & 5 & 7 & 26 & 9 & 7 & 23 & 11 & 10 & 30 & 15 & 11 & 36 & 11 \\
\hline F. Artist & 5 & 21 & 5 & 6 & 20 & 4 & 6 & 22 & 5 & 7 & 14 & 8 & 7 & 24 & 5 \\
\hline Soloist & 8 & 27 & 7 & 7 & 21 & 13 & 7 & 39 & 14 & 7 & 26 & 15 & 8 & 15 & 4 \\
\hline F. Soloist & 7 & 29 & 6 & 5 & 20 & 9 & 4 & 20 & 11 & 5 & 14 & 9 & 5 & 14 & 4 \\
\hline Principal & 7 & 42 & 12 & 9 & 27 & 13 & 8 & 23 & 8 & 9 & 20 & 8 & 8 & 23 & 6 \\
\hline PCA & 3 & 1 & 0 & 3 & 2 & 2 & 5 & 10 & 5 & 5 & 7 & 4 & 5 & 4 & 3 \\
\hline
\end{tabular}

220

App., Apprentice; F. Artist, First Artist; F. Soloist, First Soloist; PCA, Principal Character Artist; MA, Medical Attention Injury; TL Time-Loss Injury 
Injury Epidemiology in Professional Ballet

\section{2 Incidence Rates by Sex and Company Rank}

223 The incidence rates of medical attention and time-loss injuries can be found in Table 2. A 224 significant main effect of company rank was observed on medical attention injury incidence 225 rate $\left(F_{7}=2209.1 ; p<.001\right)$. Post-hoc pairwise comparisons revealed that medical attention 226 incidence rates were lower in Apprentices (2.5 per $1000 \mathrm{~h}$; 95\% Cl: 1.9-3.2) than First Soloists 227 (4.5 per $1000 \mathrm{~h} ; 95 \% \mathrm{Cl}: 3.7-5.5 ; p=.003)$, and Principals $(4.7$ per $1000 \mathrm{~h}$; $95 \% \mathrm{Cl}: 3.9-5.8$; $p=.002)$. No significant main effects of $\operatorname{sex}(p=.031)$ or sex $\times$ rank $(p=.659)$ were observed 229 on medical attention incidence rate.

230 A significant main effect of company rank was observed on time-loss injury incidence rate $\left(F_{7}\right.$ $231=1216.2 ; p<.001)$. Post-hoc pairwise comparisons revealed that Apprentices $(0.6$ per 1000 $232 \mathrm{~h} ; 95 \% \mathrm{Cl}: 0.4-1.0)$ demonstrated lower time-loss injury incidence rates than First Soloists 233 (1.5 per $1000 \mathrm{~h} ; 95 \% \mathrm{Cl}: 1.1-2.1 ; p=.015)$ and Principals $(1.7$ per $1000 \mathrm{~h} ; 95 \% \mathrm{Cl}: 1.3-2.4$; $p=.006)$. No significant main effects of $\operatorname{sex}(p=.496)$ or sex $\times$ rank $(p=.205)$ were observed on time-loss injury incidence rate. 
Injury Epidemiology in Professional Ballet

237 Table 2 Estimated marginal mean incidence rate (per $1000 \mathrm{~h}$ ), prevalence (\% injured dancers), incidence proportion (injuries per dancer) of medical attention and time-loss injuries across five consecutive seasons (95\% confidence intervals).

\begin{tabular}{|c|c|c|c|c|c|c|c|c|c|c|c|c|}
\hline & \multicolumn{6}{|c|}{ Medical Attention Injury } & \multicolumn{6}{|c|}{ Time-Loss Injury } \\
\hline & \multicolumn{2}{|c|}{ Incidence Rate } & \multicolumn{2}{|l|}{ Prevalence $^{*}$} & \multicolumn{2}{|c|}{ Incidence Proportion* } & \multicolumn{2}{|c|}{ Incidence Rate } & \multicolumn{2}{|l|}{ Prevalence $^{*}$} & \multicolumn{2}{|c|}{ Incidence Proportion* } \\
\hline & Female & Male & Female & Male & Female & Male & Female & Male & Female & Male & Female & Male \\
\hline All Ranks & $3.9(3.3-4.4)$ & $3.1(2.6-3.5)$ & $91.5(82.0-100.0)$ & $88.4(78.5-98.2)$ & $3.8(3.7-4.0)$ & $3.3(3.0-3.6)$ & $1.2(1.0-1.5)$ & $1.1(0.9-1.3)$ & $70.3(60.8-79.9)$ & $61.4(51.6-71.3)$ & $1.3(1.1-1.5)$ & $1.2(1-1.5)$ \\
\hline App. & $2.7(1.9-3.8)$ & $2.3(1.6-3.3)$ & $79.2(57.6-100.0)$ & $87.5(56.7-100.0)$ & $2.3(2.0-2.6)$ & $2.4(1.4-3.4)$ & $0.6(0.3-1.3)$ & $0.6(0.3-1.1)$ & $45.8(24.2-67.4)$ & $31.2(0.4-62.1)$ & $0.6(0.3-0.9)$ & $0.8(0.0-1.8)$ \\
\hline Artist & $3.4(2.8-4.2)$ & $3.1(2.5-3.9)$ & $94.2(79.2-100.0)$ & $93.9(70.7-100.0)$ & $4.4(3.8-4.9)$ & $3.6(3.2-4.0)$ & $0.9(0.6-1.3)$ & $1.0(0.7-1.5)$ & $68.1(53.2-83.1)$ & $73.6(50.3-96.8)$ & $1.3(0.7-1.8)$ & $1.3(0.9-1.6)$ \\
\hline F. Artist & $4.2(3.4-5.3)$ & $2.8(2.1-3.7)$ & $92.2(73.9-100.0)$ & $88.7(77.0-100.0)$ & $3.4(2.8-4.0)$ & $3.3(3.1-3.5)$ & $1.5(1.1-2.2)$ & $0.7(0.4-1.1)$ & $76.4(58.1-94.7)$ & $50.1(38.4-61.8)$ & $1.4(0.8-2.1)$ & $0.9(0.7-1.1)$ \\
\hline Soloist & $4.1(3.2-5.2)$ & $3.2(2.5-4.2)$ & $85.1(66.8-100.0)$ & $90.2(70.1-100.0)$ & $3.8(3.3-4.3)$ & $3.9(3.4-4.5)$ & $1.4(0.9-2.1)$ & $1.3(0.9-1.9)$ & $68.7(50.4-87.0)$ & $66.5(46.4-86.6)$ & $1.2(0.7-1.7)$ & $1.7(1.2-2.3)$ \\
\hline F. Soloist & $5.3(4.1-6.9)$ & $3.8(2.8-5.2)$ & 93.7 (72.9-100.0) & $100.0(88.1-100.0)$ & $4.3(3.6-5.1)$ & $4.0(3.2-4.7)$ & $1.5(0.9-2.2)$ & $1.6(1.0-2.6)$ & $71.0(50.3-91.8)$ & $82.9(71.0-94.7)$ & $1.4(0.6-2.2)$ & $1.8(1.0-2.6)$ \\
\hline Principal & $4.8(3.5-6.4)$ & $4.7(3.6-6.3)$ & $100.0(91.6-100.0)$ & $96.9(87.3-100.0)$ & $4.3(4.0-4.6)$ & $3.5(3.1-3.9)$ & $1.7(1.1-2.6)$ & $1.8(1.2-2.8)$ & $76.0(67.6-84.5)$ & $66.8(57.2-76.4)$ & $1.3(1.1-1.6)$ & $1.3(0.9-1.6)$ \\
\hline PCA & $3.2(1.8-5.5)$ & $2.1(1.3-3.5)$ & $87.5(46.4-100.0)$ & $45.0(18.1-71.9)$ & $1.8(1.0-2.7)$ & $1.1(0.7-1.5)$ & $1.6(0.7-3.6)$ & $1.1(0.5-2.1)$ & $58.3(17.2-99.4)$ & $36.7(9.7-63.6)$ & $1.2(0.3-2.0)$ & $0.6(0.2-1.0)$ \\
\hline
\end{tabular}


Injury Epidemiology in Professional Ballet

242 Intra- and Inter-Season Incidence Rates

243 A significant main effect of month $\left(F_{10}=59.7 ; p<.001\right)$ and season $\left(F_{4}=31.9 ; p<.001\right)$ was

244 observed on medical attention injury incidence rate (per $1000 \mathrm{~h}$ ); post-hoc pairwise 245 comparisons are illustrated in Figure 2. No main effects of month $(p=.029)$ or season $(p=$ $246.042)$ were observed on time-loss injury incidence rate. 
Injury Epidemiology in Professional Ballet

A
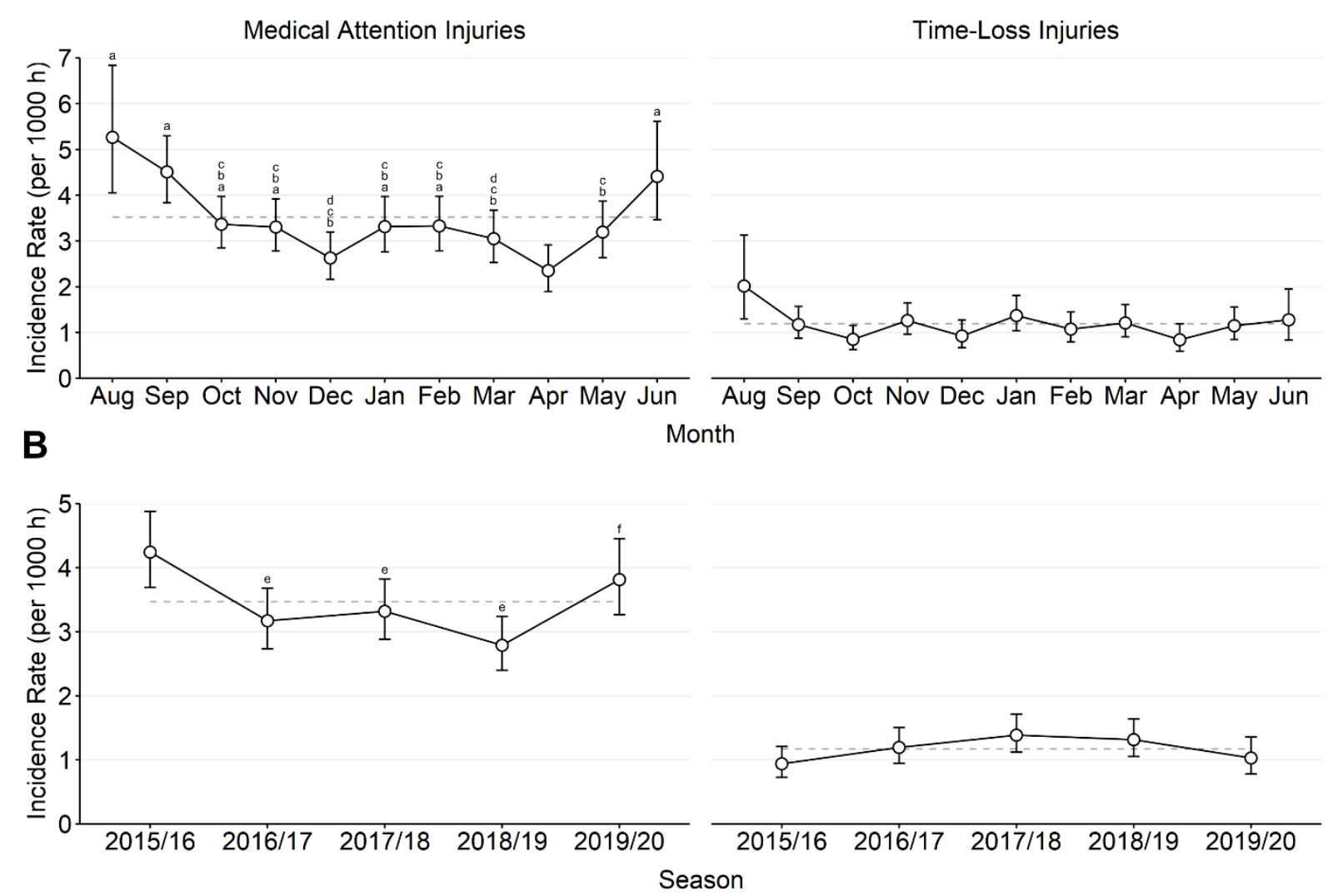

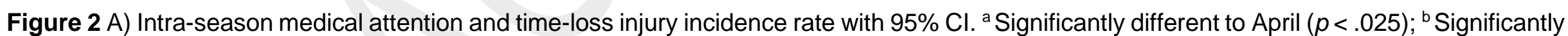
$2018 / 19$ season $(p<.025)$. 
Injury Epidemiology in Professional Ballet

252

253

254

255

256

257

258

259

260

261

262

263

264

265

266

267

268

269

270

271

\section{Incidence Rates by Production Type}

Medical attention and time-loss injury incidence rates were $6.0(95 \% \mathrm{Cl}: 5.5-6.6)$ and $2.0(95 \%$ Cl: 1.7-2.3) per 1000 hours for mixed bills and 3.7 (95\% Cl: 3.4-4.0) and 1.2 (95\% Cl: $1.1-$ 1.4) per 1000 hours for full-length productions, respectively. Medical attention and time-loss injury incidence rates were $4.2(95 \% \mathrm{Cl}$ : 3.6-4.8) and 1.5 (95\% Cl: $1.2-1.9)$ per 1000 hours for new creations and 4.3 (95\% Cl: 4.0-4.6) and $1.4(95 \% \mathrm{Cl}: 1.3-1.6)$ per 1000 hours for existing productions, respectively.

\section{Prevalence and Incidence Proportion}

Table 2 outlines the mean prevalence and incidence proportion of medical attention and timeloss injuries across the four complete seasons (2015/16-2018/19).

\section{Severity, Burden, and Aetiology of Time-loss Injuries}

Table 3 presents the median severity and percentage of time-loss injuries by severity scale. Figure 3 illustrates the time-loss injury burden by anatomical region and tissue type. The incidence rate, severity, and burden of time-loss injuries by anatomical region and tissue type are presented in Supplementary Table 1. Supplementary Table 2 outlines the percentage of medical attention and time-loss injuries by classification, occurrence, and nature. The percentage of medical attention and time-loss injuries by mechanism, activity, and footwear is provided in Supplementary Table 3. 
Injury Epidemiology in Professional Ballet

272 Table 3 Median severity of time-loss injuries and percentage of time-loss injuries by severity 273 scale (95\% confidence intervals)

\begin{tabular}{cll} 
& Female & Male \\
\hline Median Severity (days) & & \\
All Ranks & $14(10-16)$ & $14(7-16)$ \\
App. & $17(2-123)$ & $22(10-39)$ \\
Artist & $10(3-16)$ & $12(6-31)$ \\
F. Artist & $24(11-30)$ & $14(3-18)$ \\
Soloist & $9(3-33)$ & $12(3-18)$ \\
F. Soloist & $18(8-25)$ & $21(8-41)$ \\
Principal & $9(4-16)$ & $6(2-27)$ \\
PCA & $10(1-14)$ & $14(6-25)$ \\
Severity Scale (\%) & & \\
Mild (1-7 days) & $39.9(24.7-55.1)$ & $41.5(26.5-56.5)$ \\
Moderate (8-28) & $25.2(8.2-42.1)$ & $23.7(6.5-40.8)$ \\
Severe (>28) & $34.9(19.1-50.7)$ & $34.9(19.0-50.7)$
\end{tabular}

274 App., Apprentice; F. Artist, First Artist; F. Soloist, First Soloist; PCA, Principal Character Artist 
A

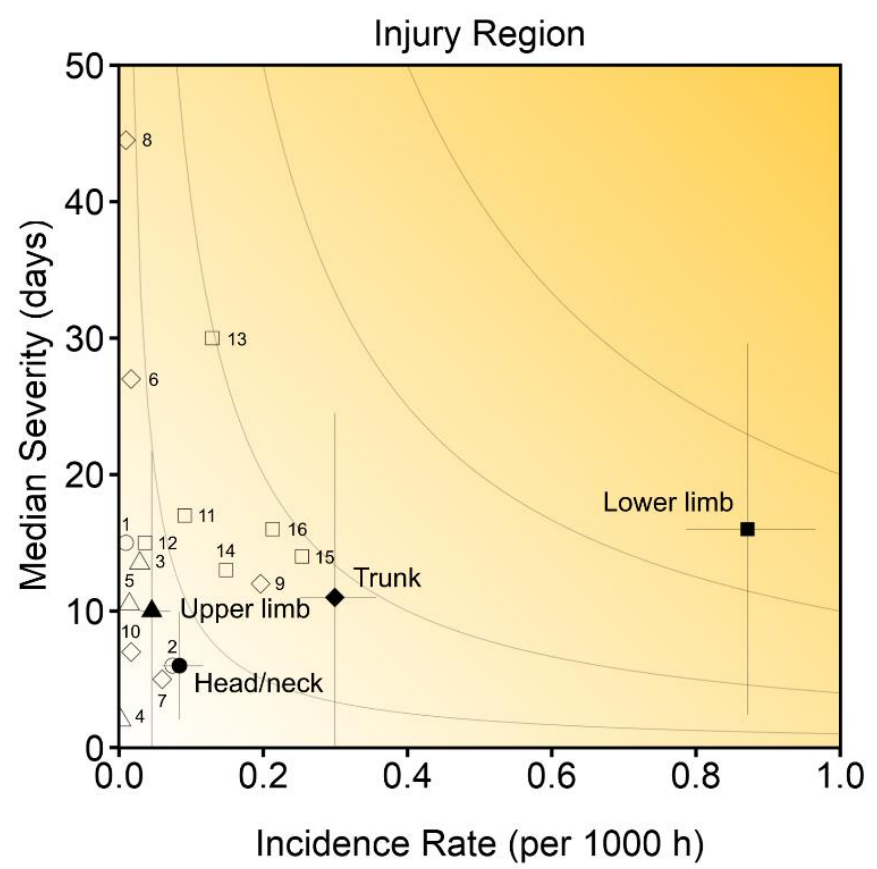

- Head/neck

1. Head

2. Neck

$\Delta$ Upper limb

3. Shoulder

$\triangle 5$. Wrist/han

$\checkmark$ Trunk

$\begin{array}{ll}\diamond & \text { 7. Thoracic spine } \quad \square \text { 15. Ankle } \\ \diamond & \text { 8. Trunk/abdomen } \\ \diamond \text { 16. Foot } \\ \diamond \text { 10. Pelvar spine } \\ - \text { Lower limb } \\ \square \text { 11. Hip/groin } \\ \square \text { 12. Thigh } \\ \square \text { 13. Knee } \\ \square \text { 14. Lower leg }\end{array}$

B

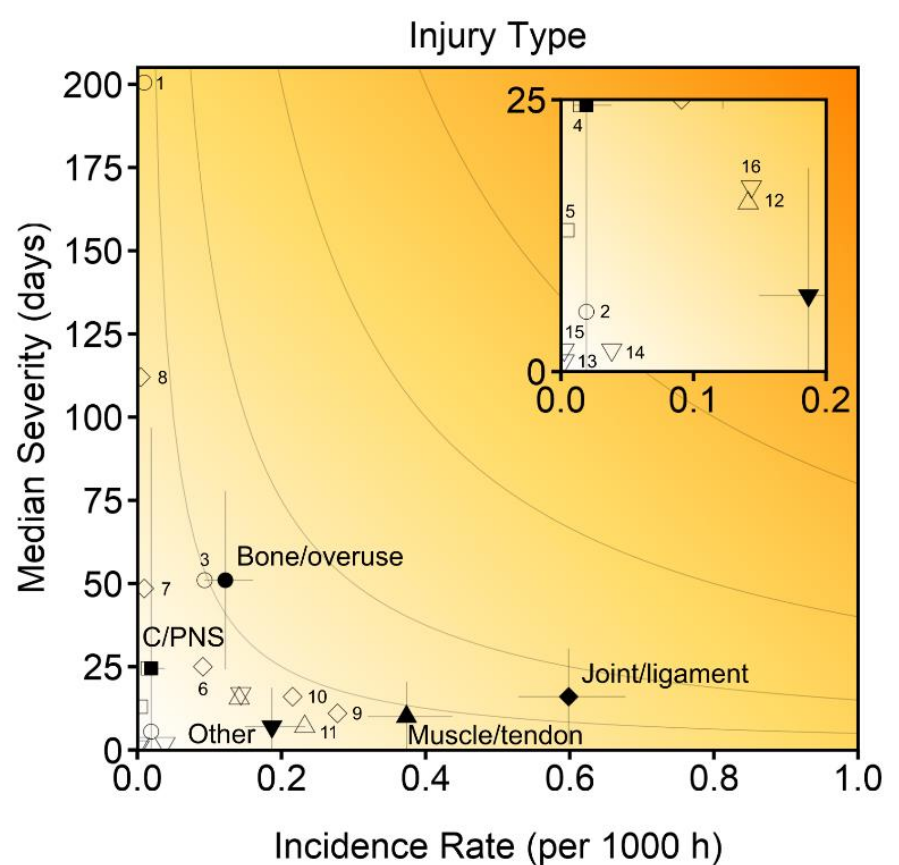

Figure 3 A) Time-loss injury burden (incidence rate $\times$ median severity) by anatomical region with 95\% Cl. B) Time-loss injury burden (incidence rate $\times$ median severity) by tissue type with $95 \% \mathrm{Cl}$. The top right corner of plot B depicts a zoomed-in subsection of the main plot identifiable by the axis. It should be noted that the $y$-axis scale across plot $A$ and $B$ are not equal. 
279 Supplementary Table 1 Number of injuries, incidence rate (injuries per $1000 \mathrm{~h}$ ), severity 280 (median days lost), and burden (days lost per $1000 \mathrm{~h}$ ) of time-loss injuries by injury region 281 and tissue type (95\% confidence intervals).

\begin{tabular}{|c|c|c|c|c|c|c|c|c|}
\hline & \multicolumn{2}{|c|}{$n$ injuries } & \multicolumn{2}{|l|}{ Incidence Rates } & \multicolumn{2}{|l|}{ Severity } & \multicolumn{2}{|l|}{ Burden } \\
\hline & Female & Male & Female & Male & Female & Male & Female & Male \\
\hline Head & 2 & 2 & $0.01(0.00-0.04)$ & $0.01(0.00-0.04)$ & $15(0-33)$ & $15(0-40)$ & $0(0-1)$ & $0(0-1)$ \\
\hline Neck & 17 & 14 & $0.08(0.05-0.12)$ & $0.07(0.04-0.12)$ & $4(0-9)$ & $6(0-13)$ & $1(0-1)$ & $1(0-1)$ \\
\hline Shoulder & 3 & 9 & $0.01(0.00-0.04)$ & $0.05(0.02-0.09)$ & $7(0-48)$ & $17(2-32)$ & $0(0-1)$ & $1(1-2)$ \\
\hline Elbow & 1 & - & $0.00(0.00-0.03)$ & - & $2(0-0)$ & - & $0(0-0)$ & - \\
\hline Wrist/hand & 1 & 5 & $0.00(0.00-0.03)$ & $0.03(0.01-0.06)$ & $14(0-0)$ & $7(0-33)$ & $0(0-0)$ & $0(0-1)$ \\
\hline Chest & 4 & 3 & $0.02(0.01-0.05)$ & $0.02(0.00-0.05)$ & $18(0-106)$ & $27(0-76)$ & $1(0-2)$ & $1(0-2)$ \\
\hline Thoracic spine & 10 & 15 & $0.04(0.02-0.08)$ & $0.08(0.05-0.13)$ & $10(0-24)$ & $4(0-32)$ & $1(0-2)$ & $2(1-3)$ \\
\hline Trunk/abdomen & 4 & - & $0.02(0.01-0.05)$ & - & $44(0-101)$ & - & $1(0-3)$ & - \\
\hline Lumbar spine & 48 & 34 & $0.22(0.16-0.29)$ & $0.17(0.12-0.24)$ & $16(2-31)$ & $5(0-37)$ & $6(5-9)$ & $6(4-9)$ \\
\hline Joint sprains & 5 & 5 & $0.02(0.01-0.05)$ & $0.03(0.01-0.06)$ & $39(0-100)$ & $6(0-39)$ & $1(1-4)$ & $1(0-1)$ \\
\hline Cartilage injury & 11 & 5 & $0.05(0.03-0.09)$ & $0.03(0.01-0.06)$ & $20(0-51)$ & $27(8-46)$ & $2(1-3)$ & $1(0-1)$ \\
\hline Synovitis, impingement, bursitis & 14 & 11 & $0.06(0.04-0.11)$ & $0.06(0.03-0.10)$ & $12(3-21)$ & $2(0-32)$ & $1(1-2)$ & $1(1-2)$ \\
\hline Muscle injury & 10 & 8 & $0.04(0.02-0.08)$ & $0.04(0.02-0.08)$ & $12(0-34)$ & $2(0-21)$ & $1(1-2)$ & $1(0-1)$ \\
\hline Pelvis/buttock & 6 & 1 & $0.03(0.01-0.06)$ & $0.01(0.00-0.04)$ & $9(0-59)$ & $1(0-0)$ & $1(0-2)$ & $0(0-0)$ \\
\hline Hip/groin & 26 & 12 & $0.12(0.08-0.17)$ & $0.06(0.03-0.11)$ & $23(0-56)$ & $10(0-24)$ & $6(4-8)$ & $1(1-2)$ \\
\hline Synovitis, impingement, bursitis & 8 & 4 & $0.04(0.02-0.07)$ & $0.02(0.01-0.05)$ & $27(0-114)$ & $15(2-28)$ & $2(1-5)$ & $0(0-1)$ \\
\hline Other injury & 7 & 3 & $0.03(0.01-0.07)$ & $0.02(0.00-0.05)$ & $33(0-92)$ & $52(21-83)$ & $2(1-4)$ & $1(0-2)$ \\
\hline Thigh & 5 & 10 & $0.02(0.01-0.05)$ & $0.05(0.03-0.10)$ & $6(0-19)$ & $16(0-38)$ & $0(0-1)$ & $1(1-2)$ \\
\hline Knee & 25 & 29 & $0.11(0.08-0.17)$ & $0.15(0.10-0.21)$ & $21(0-64)$ & $32(0-72)$ & $7(5-11)$ & $9(6-13)$ \\
\hline Joint sprains & 5 & 3 & $0.02(0.01-0.05)$ & $0.02(0.00-0.05)$ & $119(0-256)$ & $17(0-308)$ & $3(1-6)$ & $2(1-8)$ \\
\hline Tendon injury & 2 & 12 & $0.01(0.00-0.04)$ & $0.06(0.03-0.11)$ & $80(55-105)$ & $25(0-64)$ & $1(0-3)$ & $3(1-5)$ \\
\hline Lower leg & 32 & 30 & $0.14(0.10-0.20)$ & $0.15(0.11-0.22)$ & $7(0-25)$ & $18(0-48)$ & $4(3-5)$ & $7(5-10)$ \\
\hline Stress fracture & 8 & 7 & $0.04(0.02-0.07)$ & $0.04(0.02-0.08)$ & $60(23-96)$ & $71(0-143)$ & $2(1-4)$ & $3(2-7)$ \\
\hline Muscle injury & 16 & 19 & $0.07(0.04-0.12)$ & $0.10(0.06-0.15)$ & $7(0-32)$ & $14(6-22)$ & $2(1-2)$ & $2(1-2)$ \\
\hline Ankle & 66 & 40 & $0.30(0.23-0.38)$ & $0.21(0.15-0.28)$ & $14(0-42)$ & $12(0-35)$ & $13(10-17)$ & $8(6-11)$ \\
\hline Joint sprains & 21 & 6 & $0.09(0.06-0.14)$ & $0.03(0.01-0.07)$ & $14(0-38)$ & $14(0-58)$ & $3(2-5)$ & $1(1-3)$ \\
\hline Synovitis, impingement, bursitis & 20 & 14 & $0.09(0.06-0.14)$ & $0.07(0.04-0.12)$ & $22(0-85)$ & $10(0-46)$ & $5(3-8)$ & $3(2-4)$ \\
\hline Tendon injury & 19 & 14 & $0.09(0.05-0.13)$ & $0.07(0.04-0.12)$ & $7(0-43)$ & $11(0-36)$ & $3(2-5)$ & $2(1-3)$ \\
\hline Foot & 50 & 39 & $0.22(0.17-0.30)$ & $0.20(0.15-0.27)$ & $16(0-34)$ & $16(0-45)$ & $8(6-11)$ & $9(7-13)$ \\
\hline Stress fracture & 13 & 9 & $0.06(0.03-0.10)$ & $0.05(0.02-0.09)$ & $46(16-76)$ & $46(0-110)$ & $3(2-5)$ & $3(2-7)$ \\
\hline Joint sprains & 19 & 8 & $0.09(0.05-0.13)$ & $0.04(0.02-0.08)$ & $14(1-27)$ & $27(5-49)$ & $2(1-3)$ & $1(1-3)$ \\
\hline
\end{tabular}


Injury Epidemiology in Professional Ballet

283 Supplementary Table 2 Number and percentage of medical attention and time-loss injuries by classification, occurrence, and nature (95\% 284 confidence intervals).

\begin{tabular}{|c|c|c|c|c|c|c|c|c|}
\hline & \multicolumn{4}{|c|}{ Medical Attention Injury } & \multicolumn{4}{|c|}{ Time-Loss Injury } \\
\hline & \multicolumn{2}{|c|}{$n$ injuries } & \multicolumn{2}{|l|}{ Percentage } & \multicolumn{2}{|c|}{$n$ injuries } & \multicolumn{2}{|l|}{ Percentage } \\
\hline & Female & Male & Female & Male & Female & Male & Female & Male \\
\hline \multicolumn{9}{|l|}{ Classification } \\
\hline Overuse & 637 & 434 & $68.9(65.9-71.8)$ & $64.7(61.1-68.3)$ & 151 & 125 & $50.3(44.7-56.0)$ & $51.4(45.2-57.7)$ \\
\hline Traumatic & 223 & 185 & $24.1(21.4-26.9)$ & $27.6(24.2-31.0)$ & 121 & 99 & $40.3(34.8-45.9)$ & 40.7 (34.6-46.9) \\
\hline Not classified & 65 & 52 & $7.0(5.4-8.7)$ & $7.7(5.7-9.8)$ & 28 & 19 & $9.3(6.0-12.6)$ & $7.8(4.4-11.2)$ \\
\hline \multicolumn{9}{|l|}{ Occurrence } \\
\hline First episode & 597 & 427 & $64.5(61.5-67.6)$ & $63.6(60.0-67.3)$ & 213 & 162 & $71.0(65.9-76.1)$ & $66.7(60.7-72.6)$ \\
\hline Recurrence & 321 & 237 & $34.7(31.6-37.8)$ & $35.3(31.7-38.9)$ & 85 & 79 & $28.3(23.2-33.4)$ & $32.5(26.6-38.4)$ \\
\hline Not classified & 7 & 7 & $0.8(0.2-1.3)$ & $1.0(0.3-1.8)$ & 2 & 2 & $0.7(0.0-1.6)$ & $0.8(0.0-2.0)$ \\
\hline \multicolumn{9}{|l|}{ Nature } \\
\hline Extrinsic & 249 & 174 & $26.9(24.1-29.8)$ & $25.9(22.6-29.2)$ & 99 & 80 & $33.0(27.7-38.3)$ & $32.9(27.0-38.8)$ \\
\hline Intrinsic & 670 & 493 & $72.4(69.6-75.3)$ & $73.5(70.1-76.8)$ & 199 & 162 & $66.3(61.0-71.7)$ & $66.7(60.7-72.6)$ \\
\hline Not classified & 6 & 4 & $0.6(0.1-1.2)$ & $0.6(0.0-1.2)$ & 2 & 1 & $0.7(0.0-1.6)$ & $0.4(0.0-1.2)$ \\
\hline
\end{tabular}


Injury Epidemiology in Professional Ballet

286 Supplementary Table 3 Number and percentage of medical attention and time-loss injuries by injury mechanism, activity, and footwear (95\% 287 confidence intervals).

\begin{tabular}{|c|c|c|c|c|c|c|c|c|}
\hline & \multicolumn{4}{|c|}{ Medical Attention Injury } & \multicolumn{4}{|c|}{ Time-Loss Injuries } \\
\hline & \multicolumn{2}{|c|}{$n$ injuries } & \multicolumn{2}{|l|}{ Percentage } & \multicolumn{2}{|c|}{$n$ injuries } & \multicolumn{2}{|l|}{ Percentage } \\
\hline & Female & Male & Female & Male & Female & Male & Female & Male \\
\hline \multicolumn{9}{|l|}{ Mechanism } \\
\hline Jumping/landing & 200 & 206 & $21.6(19.0-24.3)$ & $30.7(27.2-34.2)$ & 81 & 92 & $27.0(22.0-32.0)$ & $37.9(31.8-44.0)$ \\
\hline Pointe & 132 & 3 & $14.3(12.0-16.5)$ & $0.4(0.0-1.0)$ & 37 & 0 & $12.3(8.6-16.1)$ & $0.0(0.0-0.0)$ \\
\hline Plié/relevé & 66 & 64 & $7.1(5.5-8.8)$ & $9.5(7.3-11.8)$ & 21 & 21 & $7.0(4.1-9.9)$ & $8.6(5.1-12.2)$ \\
\hline Lifting/lifted & 31 & 98 & $3.4(2.2-4.5)$ & $14.6(11.9-17.3)$ & 11 & 29 & $3.7(1.5-5.8)$ & $11.9(7.9-16.0)$ \\
\hline Arabesque & 65 & 20 & $7.0(5.4-8.7)$ & $3.0(1.7-4.3)$ & 15 & 7 & $5.0(2.5-7.5)$ & $2.9(0.8-5.0)$ \\
\hline Pirouette & 11 & 20 & $1.2(0.5-1.9)$ & $3.0(1.7-4.3)$ & 2 & 8 & $0.7(0.0-1.6)$ & $3.3(1.0-5.5)$ \\
\hline Non-dance related & 60 & 36 & $6.5(4.9-8.1)$ & $5.4(3.7-7.1)$ & 25 & 14 & $8.3(5.2-11.5)$ & $5.8(2.8-8.7)$ \\
\hline Cannot recall & 89 & 60 & $9.6(7.7-11.5)$ & $8.9(6.8-11.1)$ & 33 & 18 & $11.0(7.5-14.5)$ & $7.4(4.1-10.7)$ \\
\hline Not classified & 271 & 164 & $29.3(26.4-32.2)$ & $24.4(21.2-27.7)$ & 75 & 54 & $25.0(20.1-29.9)$ & $22.2(17.0-27.4)$ \\
\hline \multicolumn{9}{|l|}{ Activity } \\
\hline Rehearsal & 478 & 307 & $51.7(48.5-54.9)$ & $45.8(42.0-49.5)$ & 149 & 100 & $49.7(44.0-55.3)$ & $41.2(35.0-47.3)$ \\
\hline Performance & 206 & 110 & $22.3(19.6-25.0)$ & $16.4(13.6-19.2)$ & 66 & 45 & $22.0(17.3-26.7)$ & $18.5(13.6-23.4)$ \\
\hline Class & 104 & 140 & $11.2(9.2-13.3)$ & $20.9(17.8-23.9)$ & 34 & 49 & $11.3(7.7-14.9)$ & $20.2(15.1-25.2)$ \\
\hline Gym & 8 & 21 & $0.9(0.3-1.5)$ & $3.1(1.8-4.4)$ & 1 & 7 & $0.3(0.0-1.0)$ & $2.9(0.8-5.0)$ \\
\hline Pilates/Gyrotonics $\circledast$ & 3 & 1 & $0.3(0.0-0.7)$ & $0.1(0.0-0.4)$ & 1 & 0 & $0.3(0.0-1.0)$ & $0.0(0.0-0.0)$ \\
\hline Rehab & - & 3 & - & $0.4(0.0-1.0)$ & - & 2 & - & $0.8(0.0-2.0)$ \\
\hline Non-dance related & 56 & 39 & $6.1(4.5-7.6)$ & $5.8(4.0-7.6)$ & 25 & 20 & $8.3(5.2-11.5)$ & $8.2(4.8-11.7)$ \\
\hline Not classified & 70 & 50 & $7.6(5.9-9.3)$ & $7.5(5.5-9.4)$ & 24 & 20 & $8.0(4.9-11.1)$ & $8.2(4.8-11.7)$ \\
\hline \multicolumn{9}{|l|}{ Footwear } \\
\hline Ballet Flats & 106 & 533 & $11.5(9.4-13.5)$ & 79.4 (76.4-82.5) & 34 & 187 & $11.3(7.7-14.9)$ & $77.0(71.7-82.2)$ \\
\hline Pointe Shoes & 658 & 7 & $71.1(68.2-74.1)$ & $1.0(0.3-1.8)$ & 210 & 2 & $70.0(64.8-75.2)$ & $0.8(0.0-2.0)$ \\
\hline Character Shoes & 30 & 22 & $3.2(2.1-4.4)$ & $3.3(1.9-4.6)$ & 9 & 10 & $3.0(1.1-4.9)$ & $4.1(1.6-6.6)$ \\
\hline Barefoot & 8 & 9 & $0.9(0.3-1.5)$ & $1.3(0.5-2.2)$ & 3 & 5 & $1.0(0.0-2.1)$ & $2.1(0.3-3.8)$ \\
\hline Trainers & 20 & 22 & $2.2(1.2-3.1)$ & $3.3(1.9-4.6)$ & 6 & 7 & $2.0(0.4-3.6)$ & $2.9(0.8-5.0)$ \\
\hline Not classified & 103 & 78 & $11.1(9.1-13.2)$ & $11.6(9.2-14.0)$ & 38 & 32 & $12.7(8.9-16.4)$ & $13.2(8.9-17.4)$ \\
\hline
\end{tabular}


Injury Epidemiology in Professional Ballet

\section{DISCUSSION}

This is the first study to report longitudinal medical attention incidence rates in professional ballet. Differences in medical attention incidence rates were observed across company rank, with First Soloists and Principals demonstrating an almost two-fold greater incidence rate compared to Apprentices. The time-loss injury incidence rate observed in this study is in line with published literature, ${ }^{1-5}$ however, the severity of time-loss injures was greater, with $35 \%$ of injuries resulting in more than 28 days of modified dance activity. ${ }^{1,4}$ Consistent with previous research in professional ballet, most time-loss injuries were classified as overuse. ${ }^{1-3}$ The most common mechanism of time-loss injury was jumping and landing activities, however, a similar number of injuries did not have a clear mechanism of injury.

\section{Incidence Rate}

No studies in professional ballet have previously reported medical attention injury incidence rates, however, the values observed in the present study are similar to those seen in professional contemporary dance. ${ }^{23}$ The incidence rate of time-loss injuries in this study falls within ranges that are reported in professional ballet, ${ }^{1-3,5}$ is comparable to cricket $^{24}$ and contemporary dance, ${ }^{23}$ greater than that of modern dance, ${ }^{25,26}$ but lower than rugby union or ice hockey. 27,28 In the absence of a direct comparison of activity profiles across dance genres, it is speculative to discuss differences in incidence rates between them. While time-motion analysis has revealed reduced activity demands in contemporary dance compared to ballet, ${ }^{29}$ no such comparisons have been made between modern dance and ballet. Compared to invasion sports, however, the lower incidence rates observed in the present study may be due to fewer traumatic contact events during dance performance versus match play; incidence rates during rugby training, for example, are similar to those observed in the present study. ${ }^{30}$

First Soloists and Principals sustained between 2.0-2.2 additional medical attention injuries per 1000 hours and $0.9-1.1$ additional time-loss injuries per 1000 hours compared to 
315 Apprentices. The transition period from pre-professional training into a professional ballet 316 company has been previously identified as a potential risk factor for injury. ${ }^{31}$ Our findings, 317 however, demonstrate that Apprentices are at the lowest risk of injury compared to other company members. It is plausible that Apprentices may avoid disclosing injuries when trying to establish themselves within a new company. However, injury incidence rates are likely higher in senior ranking dancers due to the casting of more technically and physically demanding roles within these ranks compared to junior dancers. ${ }^{14,32}$ The casting of roles and distribution of workload across company ranks is, however, at the discretion of the Artistic Director, and the utilisation of junior dancers may differ across ballet companies.

Between 2.0-2.8 additional medical attention injuries per 1000 hours were observed at the start (August and September) and end (June) of the season compared to mid-season. Higher medical attention injury incidence rates at the start of a season may suggest strategies are warranted for returning dancers, such as pre-season training or a more gradual reintroduction to ballet. The higher incidence rates observed at the end of the season may be influenced by dancers who have been managing medical issues during the season. ${ }^{15}$ However, it should be noted that mixed bill productions, which demonstrate an additional 2.3 medical attention injuries and 0.8 and time-loss injuries per 1000 hours compared to full-length stand-alone productions, are more common later in the season. While inter-season differences in medical attention injury incidence rates were seen, no clear pattern was observed across the five seasons, potentially due to inter-season variation in repertoire. Understanding the incidence rates associated with production types may be beneficial to Artistic Directors and medical staff when planning and periodising a season.

\section{Severity}

The severity of time-loss injuries within the present study is almost two-fold greater than the severity previously published in professional ballet, ${ }^{1}$ similar to football, ${ }^{33}$ and lower than rugby union, ${ }^{28,30}$ and volleyball. ${ }^{34}$ Professional ballet has previously been described as a culture that 
Injury Epidemiology in Professional Ballet

normalises pain, ${ }^{15,35,36}$ which may result in dancers not reporting medical issues and dancing through discomfort. We observed that $56 \%$ of all days lost to time-loss injury were classified as 'restricted' as opposed to 'off', suggesting that dancers may still have been participating in some form of dance activity while injured.

\section{Time-Loss Injury Aetiology}

Between $65-69 \%$ of medical attention and $50-51 \%$ of time-loss injuries were insidious and a consequence of overuse. The greater proportion of overuse injuries observed under the medical attention definition suggests that overuse injuries may be underestimated using a time-loss injury definition alone. ${ }^{37}$ Previous studies in professional ballet have reported that a high proportion of time-loss injuries were overuse; $;^{1,3}$ our results align with this, although it should be noted that inter-season variation was observed. The high frequency of overuse injuries observed may also be associated with the large exposure times; the scheduled exposure hours in professional dance is greater than that reported in sport. ${ }^{14,25,34}$ The primary mechanism of time-loss injury was jumping and landing, in line with previous research. ${ }^{1} \mathrm{We}$ also observed a greater percentage of time-loss injuries associated with jumping and landing in male dancers compared to females, however, the absolute number of injuries attributed to this mechanism was similar across sexes. In contrast to sport, where injuries principally occur in competition, ${ }^{27,28,33}$ more than two-thirds of all time-loss injuries observed in the present study were attributed to training as opposed to performance. The higher proportion of trainingrelated injuries is likely due to the 3.5 fold greater exposure hours observed during class and rehearsal compared to performance. Most injuries were classified as first episodes rather than recurrences, suggesting that time-loss injury rehabilitation is largely successful. The majority of injuries were classified as intrinsic, and may therefore provide an opportunity for training interventions or appropriate load management. ${ }^{38-41}$ 
Injury Epidemiology in Professional Ballet

368

369

370

371

372

373

374

375

376

377

378

379

380

381

382

383

384

385

386

387

388

389

390

391

392

393

\section{Anatomical Region and Tissue Type}

Previous research in professional ballet has reported injury region and tissue type inconsistently, making comparison with these studies challenging. ${ }^{1,3-5}$ Generally, injuries to the distal lower extremity and joint/ligament tissue types demonstrated the greatest burden across all dancers. Ankle injuries pertaining to synovitis, impingement, and bursitis exhibited the greatest burden in female dancers, however, tendon and joint pathologies of the ankle were similar. Pointe positions, typically adopted by female dancers, require extreme range of motion of the ankle and may have negative consequences for musculoskeletal joint health. In male dancers, stress fractures to the foot and lower leg demonstrated the greatest burden. Nineteen of the twenty-one stress fractures recorded in males were attributed to jumping and landing activities and eighteen were non-traumatic. Medical management strategies addressing the joint and ligament injuries to the ankle in females and stress fractures to the foot and lower leg in males are warranted in this population. ${ }^{43}$

\section{Strengths and Limitations}

The strengths of this study include the prospective data entry from Chartered Physiotherapists, use of individualised prospectively entered dance exposure data, reporting of data through standardised entry forms, duration of data collection, consistency of the observed cohort, and the elite performance standard of the observed cohort.

Several limitations should be noted. Performance exposure was potentially inflated where individuals were allotted the total duration of a performance rather than on-stage time. Further, no register of attendance was taken for class or rehearsal, with attendance assumed but not verified. The authors, however, believe that it would be unusual for dancers to not attend scheduled dance events.

Multiple Chartered Physiotherapists were employed over the study period which may affect the uniformity of how injury data were gathered. It should be emphasised, however, that all 
Injury Epidemiology in Professional Ballet

394

395

396

397

398

399

400

401

402

403

404

405

406

407

408

409

410

411

412

413

414

415

416

physiotherapists used the same standardised entry forms and classification tools. The high frequency of overuse injuries may result in the misclassification of injury mechanism due to no traumatic inciting event. ${ }^{13}$ Data describing injury region and tissue type were only presented for time-loss injuries, which may not represent all medical attention injuries. Four injuries were rehabilitating at the point of analysis and were subsequently removed from severity calculations. Finally, one ballet company was investigated and, thus, caution should be taken when generalising findings to other companies based on the season structure, hierarchy of company ranks, and casting of featured roles across company ranks.

\section{Conclusion}

This is the first prospective cohort study to investigate the longitudinal medical attention and time-loss injury incidence rates in a professional ballet company. First Soloists and Principals experienced medical attention and time-loss injury incidence rates roughly two-fold that of Apprentices. Although no differences in intra-season time-loss injury incidence rates were observed, 2.0-2.8 additional medical attentional injuries per 1000 hours were recorded at the beginning and end of the season compared to mid-season. The majority of injuries were overuse in nature and $\sim 60 \%$ of all injuries occurred during training (rehearsal and class) compared to performance. The most common mechanism of time-loss injury was jumping and landing actions, however, many injuries were unclassified. Lower extremity injuries and injuries pertaining to joint and ligament tissue types caused the greatest burden. The results of this study may inform the design of targeted injury prevention interventions focusing on senior company ranks, intra-season variation, and jumping and landing activities in professional ballet dancers. 


\section{DISCLOSURE}

419 Competing Interests None of the authors has any conflicts of interest to declare.

420 Contributorship GR implemented the electronic data management system. All authors

421 contributed to the conception and design of the work. AM, JS, and SW completed the data

422 analysis. AM wrote the first draft and prepared all revisions. All authors reviewed and edited

423 drafts, and approved the final manuscript.

424 Acknowledgements The authors would like to acknowledge the invaluable contributions from

425 the physiotherapists and doctors who have worked with The Royal Ballet during this

426 surveillance period and recorded injury data used in this study: lan Beasley, Caryl Becker,

427 James Boyd, Pippa Carter, Judith Coe, Sarah Davies, Keith Day, Nathan Evans, Stephen

428 Garvey, Gemma Hilton, Jonathan Houghton, Kate Hutchings, Beenish Kamal, Wayne

429 Kitchener, Moira McCormack, Alexander McKinven, Karen Sheriff, Fiona Sweny, Robin

430 Vellosa, and Daniel Watson. The authors would also like to acknowledge the significant

431 contributions of Philip Mosley for inputting dance exposure data throughout the study period.

432 Funding No funding was provided for this study

433 Ethical Approval St Mary's University, Twickenham Ethics Board, in accordance with the 434 Declaration of Helsinki.

435 Data Sharing No data are available

436 Patient Involvement There was no patient or public involvement in this study 
Injury Epidemiology in Professional Ballet

\section{REFERENCES}

1. Allen N, Nevill A, Brooks J, Koutedakis Y, Wyon M. Ballet injuries: Injury incidence and severity over 1 year. J Orthop Sports Phys Ther. 2012;42(9):781-790. doi:10.2519/jospt.2012.3893

2. Allen N, Nevill AM, Brooks JHM, Koutedakis Y, Wyon MA. The effect of a comprehensive injury audit program on injury incidence in ballet: A 3-year prospective study. Clin J Sport Med. 2013;23(5):373-378. doi:10.1097/JSM.0b013e3182887f32

3. Nilsson C, Leanderson J, Wykman A, Strender LE. The injury panorama in a Swedish professional ballet company. Knee Surgery, Sport Traumatol Arthrosc. 2001;9(4):242246. doi:10.1007/s001670100195

4. Byhring S, Bø K. Musculoskeletal injuries in the Norwegian National Ballet: A prospective cohort study. Scand J Med Sci Sport. 2002;12(6):365-370. doi:10.1034/j.1600-0838.2002.01262.x

5. Ramkumar PN, Farber J, Arnouk J, Varner KE, Mcculloch PC. Injuries in a Professional Ballet Dance Company A 10-year Retrospective Study. J Danc Med Sci. 2016;20(1):30-37. doi:10.12678/1089-313X.20.1.30

6. Garrick JG, Requa RK. An analysis of epidemiology and financial outcome. Am J Sports Med. 1993;21(4):586-590. doi:10.1177/036354659302100417

7. Solomon R, Micheli L. The "cost" of injuries in a professional ballet company: five-year study. Med Probl Perform Art. 1995;64:164-170.

8. Clarsen B, Bahr R. Matching the choice of injury/illness definition to study setting, purpose and design: One size does not fit all! Br J Sports Med. 2014;48(7):510-512. doi:10.1136/bjsports-2013-093297

9. Fuller CW, Ekstrand J, Junge A, et al. Consensus statement on injury definitions and data collection procedures in studies of football (soccer) injuries. Scand J Med Sci 
Injury Epidemiology in Professional Ballet

Sport. 2006;16(2):83-92. doi:10.1111/j.1600-0838.2006.00528.x

10. Fuller CW, Molloy MG, Bagate C, et al. Consensus statement on injury definitions and data collection procedures for studies of injuries in rugby union. Br J Sports Med. 2007;41(5):328-331. doi:10.1136/bjsm.2006.033282

11. Tallent J, de Weymarn C, Ahmun R, Jones TW. The impact of all-rounders and team injury status on match and series success in international cricket. J Sports Sci. 2020;00(00):1-4. doi:10.1080/02640414.2020.1798721

12. Finch C. A new framework for research leading to sports injury prevention. J Sci Med Sport. 2006;9(1-2):3-9. doi:10.1016/j.jsams.2006.02.009

13. Bahr R, Clarsen B, Derman W, et al. International Olympic Committee consensus statement: Methods for recording and reporting of epidemiological data on injury and illness in sport 2020 (including STROBE Extension for Sport Injury and Illness Surveillance (STROBE-SIIS)). Br J Sports Med. 2020;54(7):372-389. doi:10.1136/bjsports-2019-101969

14. Kozai AC, Twitchett E, Morgan S, Wyon MA, Wyon MA. Workload Intensity and Rest Periods in Professional Ballet: Connotations for Injury. Int J Sports Med. 2020;41(6):373-379. doi:10.1055/a-1083-6539

15. Bolling C, van Rijn RM, Pasman HR, van Mechelen W, Stubbe JH. In your shoes: A qualitative study on the perspectives of professional dancers and staff regarding dance injury and its prevention. Trans/ Sport Med. 2021;00:1-9. doi:10.1002/tsm2.226

16. Rae K, Orchard J. The Orchard Sports Injury Classification System (OSICS) version 10. Clin J Sport Med. 2007;17(3):201-204. doi:10.1097/JSM.0b013e318059b536

17. Alonso JM, Edouard P, Fischetto G, Adams B, Depiesse F, Mountjoy M. Determination of future prevention strategies in elite track and field: Analysis of Daegu 2011 IAAF Championships injuries and illnesses surveillance. Br J Sports Med. 
Injury Epidemiology in Professional Ballet 2012;46(7):505-514. doi:10.1136/bjsports-2012-091008

18. Bere T, Alonso JM, Wangensteen A, et al. Injury and illness surveillance during the 24th Men's Handball World Championship 2015 in Qatar. Br J Sports Med. 2015;49(17):1151-1156. doi:10.1136/bjsports-2015-094972

19. Roos KG, Marshall SW. Definition and usage of the term "overuse injury" in the us high school and collegiate sport epidemiology literature: A systematic review. Sport Med. 2014;44(3):405-421. doi:10.1007/s40279-013-0124-z

20. Bates D, Mächler M, Bolker BM, Walker SC. Fitting linear mixed-effects models using Ime4. J Stat Softw. 2015;67(1):1-48. doi:10.18637/jss.v067.i01

21. Fox J, Weisberg S. An R Companion to Applied Regression. Third edit. Thousand Oaks, CA: Sage; 2019. https://socialsciences.mcmaster.ca/jfox/Books/Companion/.

22. Lenth R. emmeans: Estimated Marginal Means, aka Least-Squares Means. 2020. https://cran.r-project.org/package=emmeans.

23. Jeffries AC, Wallace L, Coutts AJ, Cohen AM, McCall A, Impellizzeri FM. Injury, Illness, and Training Load in a Professional Contemporary Dance Company: A Prospective Study. J AthI Train. 2020;55(9):967-976. doi:10.4085/1062-6050-477-19

24. Orchard J, James T, Alcott E, Carter S, Farhart P. Injuries in Australian cricket at first class level 1995/1996 to 2000/2001. Br J Sports Med. 2002;36(4):270-275. doi:10.1136/bjsm.36.4.275

25. Bronner S, McBride C, Gill A. Musculoskeletal injuries in professional modern dancers: a prospective cohort study of 15 years. J Sports Sci. 2018;36(16):18801888. doi:10.1080/02640414.2018.1423860

26. Bronner S, Wood L. Impact of touring, performance schedule, and definitions on 1year injury rates in a modern dance company. J Sports Sci. 2017;35(21):2093-2104. doi:10.1080/02640414.2016.1255772 
Injury Epidemiology in Professional Ballet

27. McKay CD, Tufts RJ, Shaffer B, Meeuwisse WH. The epidemiology of professional ice hockey injuries: A prospective report of six NHL seasons. Br J Sports Med. 2014;48(1):57-62. doi:10.1136/bjsports-2013-092860

28. Brooks JHM, Fuller CW, Kemp SPT, Reddin DB. Epidemiology of injuries in English professional rugby union: Part 1 match injuries. Br J Sports Med. 2005;39(10):757766. doi:10.1136/bjsm.2005.018135

29. Wyon M, Twitchett E, Angioi M, Clarke F, Metsios G, Koutedakis Y. Time motion and video analysis of classical ballet and contemporary dance performance. Int J Sports Med. 2011;32(11):851-855. doi:10.1055/s-0031-1279718

30. Brooks JHM, Fuller CW, Kemp SPT, Reddin DB. Epidemiology of injuries in English professional rugby union: Part 2 training injuries. Br J Sports Med. 2005;39(10):767775. doi:10.1136/bjsm.2005.018408

31. Fuller M, Moyle GM, Hunt AP, Minett GM. Ballet and Contemporary Dance Injuries When Transitioning to Full-Time Training or Professional Level Dance: A Systematic Review. J Danc Med Sci. 2019;23(3):112-125. doi:10.12678/1089-313X.23.3.112

32. Twitchett E, Angioi M, Koutedakis $\mathrm{Y}$, Wyon M. The demands of a working day among female professional ballet dancers. J Danc Med Sci. 2010;14(4):127-132.

33. Hawkins RD, Fuller CW. A prospective epidemiological study of injuries in four English professional football clubs. Br J Sports Med. 1999;33(3):196-203. doi:10.1136/bjsm.33.3.196

34. Bahr R, Reeser JC. Injuries among World-Class Professional Beach Volleyball Players. Am J Sports Med. 2003;31(1):119-125. doi:10.1177/03635465030310010401

35. McEwen K, Young K. Ballet and pain: Reflections on a risk-dance culture. Qual Res Sport Exerc Heal. 2011;3(2):152-173. doi:10.1080/2159676X.2011.572181

36. Wainwright SP, Williams C, Turner BS. Fractured identities: Injury and the balletic 
Injury Epidemiology in Professional Ballet body. Health (Irvine Calif). 2005;9(1):49-66. doi:10.1177/1363459305048097

37. Owoeye OBA, Ghali B, Befus K, et al. Epidemiology of all-complaint injuries in youth basketball. Scand J Med Sci Sport. 2020;30(12):2466-2476. doi:10.1111/sms.13813

38. Soligard T, Schwellnus M, Alonso J-M, et al. How much is too much? (Part 1) International Olympic Committee consensus statement on load in sport and risk of injury. Br J Sports Med. 2016;50(17):1030-1041. doi:10.1136/bjsports-2016-096581

39. Schwellnus M, Soligard T, Alonso JM, et al. How much is too much? (Part 2) International Olympic Committee consensus statement on load in sport and risk of illness. Br J Sports Med. 2016;50(17):1043-1052. doi:10.1136/bjsports-2016-096572

40. Lisman P, De La Motte S, Gribbin T, Jaffin D, Murphy K, Deuster P. A systematic review of the association between physical fitness and musculoskeletal injury risk: Part 1-Cardiorespiratory endurance. J Strength Cond Res. 2017;31(11):3218-3234. doi:10.1519/jsc.0000000000002174

41. De La Motte SJ, Gribbin TC, Lisman P, Murphy K, Deuster PA. Systematic review of the association between physical fitness and musculoskeletal injury risk: Part 2 Muscular endurance and muscular strength. J Strength Cond Res. 2017;31(11):32183234. doi:10.1519/JSC.0000000000002174

42. Russell J a, Shave RM, Yoshioka H, Kruse DW, Koutedakis Y, Wyon M a. Magnetic resonance imaging of the ankle in female ballet dancers en pointe. Acta radiol. 2010;51(6):655-661. doi:10.3109/02841851.2010.482565

43. Buckthorpe M, Wright S, Bruce-Low S, et al. Recommendations for hamstring injury prevention in elite football: Translating research into practice. $\mathrm{Br} J$ Sports Med. 2019;53(7):449-456. doi:10.1136/bjsports-2018-099616 